ARTICLE

\title{
An extended APOBEC3A mutation signature in cancer
}

\author{
Adam Langenbucher ${ }^{1,9}$, Danae Bowen (10) 2,9, Ramin Sakhtemani (1) 1,3,4,9, Elodie Bournique (1) 2, \\ Jillian F. Wise ${ }^{1,3,5,6}$, Lee Zou (10 ${ }^{1,3 凶}$, Ashok S. Bhagwat (10) ${ }^{4,7 凶}$, Rémi Buisson (1) ${ }^{2,8 凶} \&$ \\ Michael S. Lawrence (i) 1,3,5凶
}

APOBEC mutagenesis, a major driver of cancer evolution, is known for targeting $T p C$ sites in DNA. Recently, we showed that APOBEC3A (A3A) targets DNA hairpin loops. Here, we show that DNA secondary structure is in fact an orthogonal influence on $A 3 A$ substrate optimality and, surprisingly, can override the $T p C$ sequence preference. $V p C$ (non- $T p C$ ) sites in optimal hairpins can outperform TpC sites as mutational hotspots. This expanded understanding of APOBEC mutagenesis illuminates the genomic Twin Paradox, a puzzling pattern of closely spaced mutation hotspots in cancer genomes, in which one is a canonical $\mathrm{TpC}$ site but the other is a $\mathrm{VpC}$ site, and double mutants are seen only in trans, suggesting a two-hit driver event. Our results clarify this paradox, revealing that both hotspots in these twins are optimal A3A substrates. Our findings reshape the notion of a mutation signature, highlighting the additive roles played by DNA sequence and DNA structure.

\footnotetext{
${ }^{1}$ Massachusetts General Hospital Cancer Center, Harvard Medical School, Boston, MA, USA. ${ }^{2}$ Department of Biological Chemistry, Center for Epigenetics and Metabolism, Chao Family Comprehensive Cancer Center, School of Medicine, University of California Irvine, Irvine, CA, USA. ${ }^{3}$ Department of Pathology, Massachusetts General Hospital, Harvard Medical School, Boston, MA, USA. ${ }^{4}$ Department of Chemistry, Wayne State University, Detroit, MI, USA. ${ }^{5}$ Broad Institute of Harvard and MIT, Cambridge, MA, USA. ${ }^{6}$ Department of Cancer Immunology, Institute for Cancer Research, University of Oslo, Oslo, Norway. ${ }^{7}$ Department of Biochemistry, Microbiology and Immunology, Wayne State University School of Medicine, Detroit, MI, USA. ${ }^{8}$ Department of Pharmaceutical Sciences, School of Pharmacy and Pharmaceutical Sciences, University of California Irvine, Irvine, CA, USA. ${ }^{9}$ These authors contributed equally: Adam Langenbucher, Danae Bowen, Ramin Sakhtemani.凶email: Izou1@mgh.harvard.edu; axb@chem.wayne.edu; rbuisson@uci.edu; mslawrence@mgh.harvard.edu
} 
nderstanding the global and local genomic preferences of mutational processes enables elucidation of the biological history of tumors and helps to tell apart recurrent driver mutations that confer a fitness advantage from passenger mutations that are caused by predictable mutational mechanisms. Mutagenic processes in cancer leave DNA footprints called mutation signatures ${ }^{1}$, typically characterized by the type of basepair substitution (e.g., C:G to T:A), and the immediate flanking sequence context (e.g., trinucleotide). Mutation signatures have been crucially important in revealing a variety of endogenous and exogenous mutational processes in cancer, and have been useful in uncovering the biological origins and natural history of numerous tumor types. One of the most prominent mutation signatures in cancer, present in over half of human tumors, is called the APOBEC signature, or Signatures 2/13, and derives from the activity of the APOBEC3A (A3A) and APO$\mathrm{BEC} 3 \mathrm{~B}$ (A3B) cytosine deaminases, which preferentially deaminate cytosines in the context of an immediately preceding thymine (written $\mathrm{TpC})^{1,2}$. A rush of recent reports has detailed the crucial importance of this signature in driving tumorigenesis and facilitating the emergence of drug resistance ${ }^{3-6}$. The APO$\mathrm{BEC}$ mutation signature was originally established based on the ability of A3A/A3B to deaminate cytosines in single-stranded unstructured DNA substrates ${ }^{7,8}$ or in short viral sequences ${ }^{9}$, and it has long been the established framework through which APOBEC3 mutagenesis is viewed. The characterization of APOBEC3 activity informed by unstructured templates has led to this family of enzymes being commonly referred to as the TC-specific APOBECs ${ }^{10-13}$, their deaminase activity defined as being TCdependent ${ }^{14}$, and their mutational signature described simply as "the tell-tale $5^{\prime}-\mathrm{TpC}$ signature"15. This framework for understanding the APOBEC mutation signature(s) has proven useful in analyzing and explaining much of the endogenous APOBEC mutational landscape in cancer.

While the preference of A3A/A3B for the TC sequence motif is evident in tumors, structural features are also crucial to consider 16,17 . Hairpin structures assumed by $\mathrm{RNA}^{18,19}$, or by DNA $^{20,21}$ while it is transiently single-stranded, e.g., during replication, can greatly increase their fitness as A3A substrates. Furthermore, the activity of $\mathrm{A} 3 \mathrm{~A}$ at these hairpin loci can be reliably predicted from features of the hairpin, namely stem strength, loop length, and the positioning of the $\mathrm{TpC}$ site within the loop. In particular, TpC sites at the $3^{\prime}$-end of triloops (3-nt loops) in very stably paired stem-loops were found to be hundreds of times more mutable than comparable linear substrates. This explained numerous recurrent mutation hotspots in cancer genomes (previously assumed to be driver events) as instead merely recurrent passenger events arising at perfect $\mathrm{A} 3 \mathrm{~A}$ substrates.

A particular class of twin mutation hotspots in APOBEC+ samples has eluded explanation, a puzzle that we refer to as the genomic Twin Paradox. In these twin pairs, one hotspot is clearly a perfect $\mathrm{A} 3 \mathrm{~A}$ substrate (suggesting it may be an $\mathrm{A} 3 \mathrm{~A}$-induced passenger event), but the other hotspot is a non-TpC site (suggesting it is not an APOBEC substrate at all). Initial reports ${ }^{22,23}$ of these sites noted that they are separated by just 1 or 2 basepairs, are flanked by palindromic sequences, and are recurrently mutated in APOBEC+ tumors. An example occurs in the promoter of the gene PLEKHS1, where a pair of highly mutated hotspots are separated by just two basepairs. The fact that these mutations are located in a gene promoter lends weight to the idea that they might have a functional role. Indeed, recent work ${ }^{24,25}$ has focused on these twin hotspots mutations in PLEKHS1 and ADGRG6 (also called GPR126) as potential drivers or biomarkers of bladder cancer. In some cases, a pair of twin hotspots is so frequently mutated that we are able to observe some patients carrying mutations at both sites. These rare double-mutated tumors allow us an opportunity to observe whether the mutations occur on the same DNA allele (in cis), or on different alleles (in trans). Surprisingly, the mutations have been seen in trans in all observed cases $^{23}$, an intriguing pattern that suggests a possible double-hit tumor suppressor mechanism, in which cancer cells achieve a fitness benefit from inactivating both copies of the gene. However, whether the twin hotspots are both drivers, or both passengers, or one of each, has remained a mystery.

Recognizing the important effect of DNA secondary structure on $\mathrm{A} 3 \mathrm{~A}$ substrate optimality, here we set out to revisit the traditional definition of the APOBEC mutation signature, this time agnostic of primary sequence, and without assuming that $\mathrm{A} 3 \mathrm{~A}$ acts on unstructured substrates. Our analyses reveal that $\mathrm{VpC}$ substrates $(\mathrm{V}=\operatorname{not} \mathrm{T})$, when presented in an optimal hairpin context, can be excellent substrates for $\mathrm{A} 3 \mathrm{~A}$, superior to many TpC substrates. Furthermore, we show that mutations at $\mathrm{VpC}$ sites in optimal DNA hairpins are highly enriched among mutations in $\mathrm{A} 3 \mathrm{~A}$-positive cancers. We see this $\mathrm{VpC}$ hairpin signature as completing an extended APOBEC3A signature. Finally, we are able to resolve the genomic Twin Paradox, by revealing that actually both sites in the twin hotspots are structurally optimal $\mathrm{A} 3 \mathrm{~A}$ substrates, suggesting that these twin mutation hotspots are likely twin passenger events due to $\mathrm{A} 3 \mathrm{~A}$ activity.

\section{Results}

APOBEC3A is capable of deaminating $\mathrm{VpC}$ sites in DNA hairpins in vitro. To systematically investigate the deamination activity of APOBEC3A on a variety of structured and unstructured substrates, we incubated extracts of APOBEC3A-expressing cells with a panel of DNA oligos and measured the catalytic activity of $\mathrm{A} 3 \mathrm{~A}$ in vitro ${ }^{20}$. Previous reports ${ }^{1,2,10-15,26}$ have established A3A's strong preference for a $5^{\prime}$-T over all other preceding nucleotides when the target substrate is singlestranded. Our results confirm this: for example, an unstructured (non-hairpin) DNA substrate with a $5^{\prime}$-TC sequence showed moderate activity in the activity assay, but changing the $5^{\prime}$-TC to a 5'-AC abolished activity (Fig. 1). Furthermore, corroborating our previous work, A3A shows markedly stronger activity when the $\mathrm{TpC}$ is presented at the $3^{\prime}$-end of a short hairpin loop closed by a stably base-paired stem. This hairpin TpC site is a highly optimal substrate for $\mathrm{A} 3 \mathrm{~A}$, as we previously reported ${ }^{20}$. However, we were surprised to see that when we tested a $5^{\prime}-\mathrm{AC}$ in the hairpin context, A3A showed significant and highly reproducible enzymatic activity, even higher than the activity on the non-hairpin 5'-TC (Fig. 1). We tested additional VpC hairpin sites and observed similar levels of activity (Supplementary Fig. 1A). These surprising results suggest orthogonal, compounding influences of having an optimal primary structure (local DNA sequence) and secondary structure (DNA hairpin) on A3A activity. Finally, to verify that A3A activity is truly the ratelimiting step in our in vitro assay and that the difference in A3A activity at DNA hairpins is not the result of differential activity of uracil DNA glycosylase (UDG) modulated by the structures of these substrates, we tested synthetic uracil-containing DNA substrates in the cleavage assay. Both a non-hairpin substrate and a hairpin substrate were cleaved quantitatively under the assay conditions (Supplementary Fig. 1B), confirming that our assay is not rate-limited by UDG activity, and provides a faithful readout of APOBEC activity levels.

APOBEC + tumors accumulate mutations at $\mathrm{VpC}$ sites in hairpin-forming sequences. The ability of $\mathrm{A} 3 \mathrm{~A}$ to deaminate non-TpC sites in DNA hairpins in vitro prompted us to reinvestigate whether $\mathrm{C} \rightarrow(\mathrm{T} / \mathrm{G})$ mutations accumulate at non-TpC 


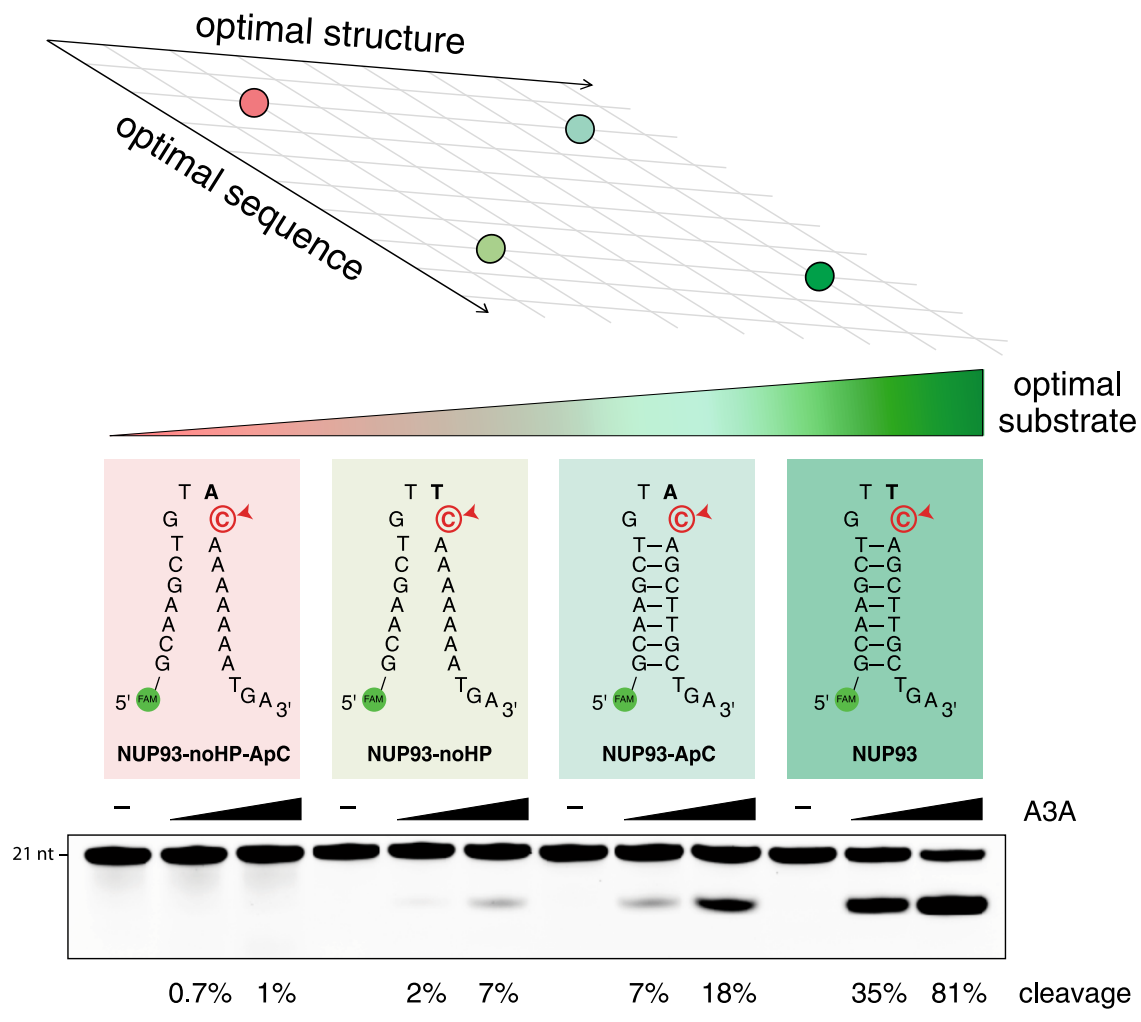

Fig. 1 Primary sequence and secondary structure contribute additively to APOBEC3A substrate optimality. Four substrates are compared, each a version of the DNA hairpin site in the human NUP93 gene. In vitro APOBEC3A activity assay shows that APOBEC3A substrate optimality increases as the DNA sequence improves in optimality (changing an $\mathrm{ApC}$ site to a $\mathrm{TpC}$ site), and also as the DNA structure improves in optimality (changing from a nonhairpin site to a hairpin site). Strongest activity is seen when both sequence and structure are optimal. Source data are provided as a Source Data file.

sites in hairpin-forming sequences in patients with high levels of APOBEC mutations. We analyzed a collection of published whole-genome and whole-exome-sequenced tumors from a variety of cancer types and assessed their mutational spectra and mutation rates in various genomic contexts. We concentrated on a set of patients with strong evidence of APOBEC-mediated mutational histories, using non-negative matrix factorization (NMF) to decompose mutation signatures 1,2 and defining as APOBEC+ those tumors with at least $50 \%$ of their mutations assigned to the APOBEC mutation signature. We aggregated mutation statistics across disparate genomic sites sharing secondary structure characteristics regardless of the preceding nucleotide, in order to explore how hairpin-feature dependency of $\mathrm{A} 3 \mathrm{~A}$ mutability at $\mathrm{TpC}$ sites can be extended to $\mathrm{VpC}$ contexts as well. We showed in our previous work that APOBEC3A mutation frequency at $\mathrm{TpC}$ sites increases with hairpin stem strength (here defined as \#AT basepairs $+3 \times$ \#GC basepairs). In our broadened analysis across all cytosines, we observed that this dependency holds true for $\mathrm{VpC}$ sites as well (Fig. 2A). We also observed the same preferences for smaller loop sizes and 3'positioning within the loop for $\mathrm{VpC}$ sites as for TpC's (Fig. 2B). Notably, all three possible $\mathrm{VpC}$ sequences (ApC, $\mathrm{CpC}, \mathrm{GpC}$ ) showed increasing mutation rate in strong hairpins (Supplementary Fig. $2 \mathrm{~A}$ ), and all 16 possible NNC triloops demonstrated the effect (Supplementary Fig. 2B). Overall, TpC sites have higher mutation rates than $\mathrm{VpC}$ sites in the same structural context. However, VpC sites in optimal hairpins displayed significantly higher mutation rates than TpC sites in linear DNA or suboptimal hairpins. These results confirm that the activity of A3A on a particular DNA substrate cannot be predicted simply by looking at the identity of the $5^{\prime}$ base, but instead both the primary and secondary structures must be considered.
To further investigate whether A3A could be responsible for $\mathrm{VpC}$ hairpin mutations in tumors, we tested a variety of additional $\mathrm{TpC}$ and $\mathrm{VpC}$ sites in our in vitro assay for $\mathrm{A} 3 \mathrm{~A}$ activity and showed that both classes of sites include examples of both good and poor substrates for A3A (Fig. 2C). Hairpins with a $\mathrm{VpC}$ sequence can be better substrates for $\mathrm{A} 3 \mathrm{~A}$ than linear substrates with a $\mathrm{TpC}$ sequence, or hairpins with a unfavorably positioned $\mathrm{TpC}$ sequence. Nevertheless, $\mathrm{TpC}$ sequences are always better substrates for $\mathrm{A} 3 \mathrm{~A}$ than $\mathrm{VpC}$ sequences in the same structural context.

Together, our computational analysis of the $\mathrm{VpC}$ mutations in APOBEC + tumors and our in vitro biochemical studies suggest that a $5^{\prime}$-situated thymine is not absolutely necessary for making an $\mathrm{A} 3 \mathrm{~A}$ substrate. The strong structural preference of $\mathrm{A} 3 \mathrm{~A}$ for cytosines in optimal hairpins may override the sequence preference for $\mathrm{TpC}$ in vitro and in tumors.

Notably, many $\mathrm{C} \rightarrow \mathrm{T}$ mutations in $\mathrm{VpC}$ hairpins were previously classified as due to the Aging signature ${ }^{1}$. Our results suggest that some of these mutations in APOBEC+ tumors arise from APOBEC activity. We computed the percent of $\mathrm{C} \rightarrow \mathrm{T}$ mutations at VCG sequences in each tumor that were in highly optimal hairpins. This was usually low $(<1 \%)$, but was elevated in a minority of tumors, reaching as high as 5\% (Supplementary Fig. $3 \mathrm{~A}$ ). Of the 36 tumors above $1 \%$, half were breast cancers, followed by five bladder tumors and three lung adenocarcinomas. These three tumor types are among the most highly APOBECenriched tumor types, supporting APOBEC mutagenesis as their source. Some of them have elevated expression of APOBEC3A and/or APOBEC3B, however some do not (Supplementary Fig. 3B). We showed recently ${ }^{18}$ that the APOBEC mutation signature is a poor predictor of $\mathrm{APOBEC} 3 \mathrm{~A} / \mathrm{B}$ expression in tumors, because while APOBEC mutations are a permanent 

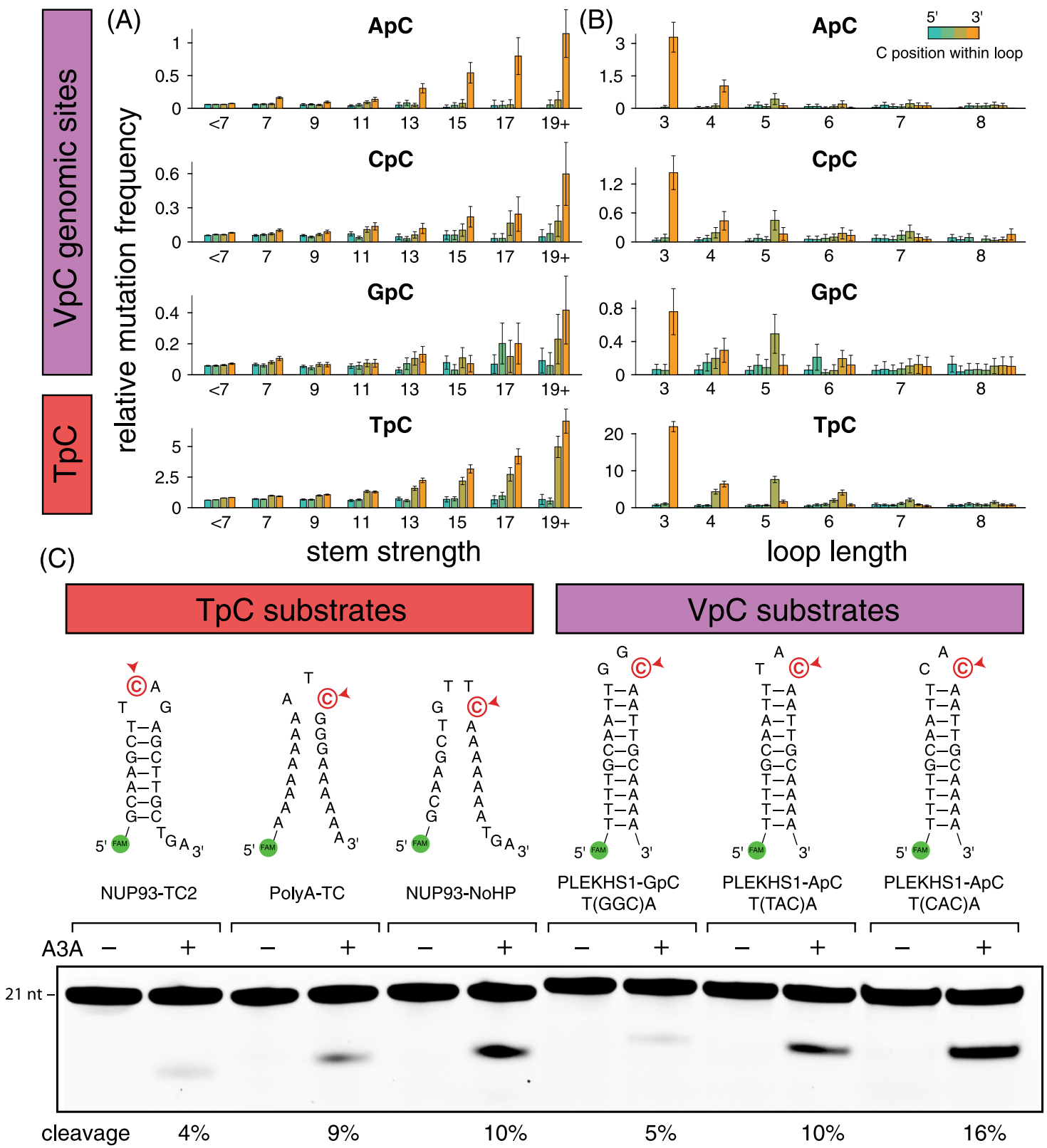

Fig. 2 VpC sites and TpC sites show similar patterns of mutability. A Relative mutation frequency in APOBEC + tumor samples increases with strength of hairpin base-pairing potential, both for $\mathrm{TpC}$ genomic sites, as well as for $\mathrm{ApC}, \mathrm{CpC}$, and $\mathrm{GpC}$ genomic sites (collectively known as $\mathrm{VpC}$ sites). Error bars represent $95 \%$ confidence intervals. B Mutation frequency decreases with size of hairpin loop, and requires optimal positioning of the cytosine within the loop, both for $\mathrm{TpC}$ and $\mathrm{VpC}$ sites. Error bars represent $95 \%$ confidence intervals. C In vitro APOBEC3A activity assay shows that APOBEC3A substrate optimality is jointly determined by sequence and structure, both for $\mathrm{TpC}$ and $\mathrm{VpC}$ sites. Source data are provided as a Source Data file.

record in a cancer cell's genome, APOBEC expression can be transient, returning to baseline after mutagenic episodes 4 .

Analyzing mutational strand asymmetry, we and others previously ${ }^{27-30}$ reported that APOBEC-associated TpC mutations are enriched on the replicative lagging-strand template (LGST), in comparison to the leading-strand template (LDST), suggesting that they occur during DNA replication. In contrast, there was little bias between the transcribed and non-transcribed DNA strands in transcribed regions of the genome. We repeated this analysis on APOBEC-associated VpC mutations (Supplementary Fig. 4) and found that they exhibited the same association with replication, but no associations with transcription or fragile sites $^{31}$, the same pattern as APOBEC-associated TpC mutations, suggesting that both $\mathrm{TpC}$ and $\mathrm{VpC}$ mutations arise primarily during DNA replication.
Mutations at $\mathrm{VpC}$ hairpin sites are due to APOBEC3A not APOBEC3B. Having demonstrated the increased mutation rates of $\mathrm{VpC}$ hairpins in APOBEC+ tumors and the ability of A3A to modify $\mathrm{VpC}$ hairpins in vitro, we next asked whether $\mathrm{A} 3 \mathrm{~A}$ is the main driver of $\mathrm{VpC}$ mutations in hairpins in tumors. We previously ${ }^{20}$ characterized APOBEC+ tumors by their A3A vs. A3B character, using an established metric ${ }^{32}$. This yields a Yshaped "bird plot" (Fig. 3A) that separates A3B-dominated tumors (left wing) from A3A-dominated tumors (right wing) from APOBEC-negative tumors (body). Mutations at TpC sites in hairpins are enriched in A3A-dominated tumors (red dots, Fig. 3B). Similarly, mutations at $\mathrm{VpC}$ sites in hairpins are also enriched in A3A-dominated tumors (magenta dots, Fig. 3C). Strikingly, no enrichment of mutations in hairpins is seen in A3B-dominated tumors, either for $\mathrm{TpC}$ hairpins (as previously 


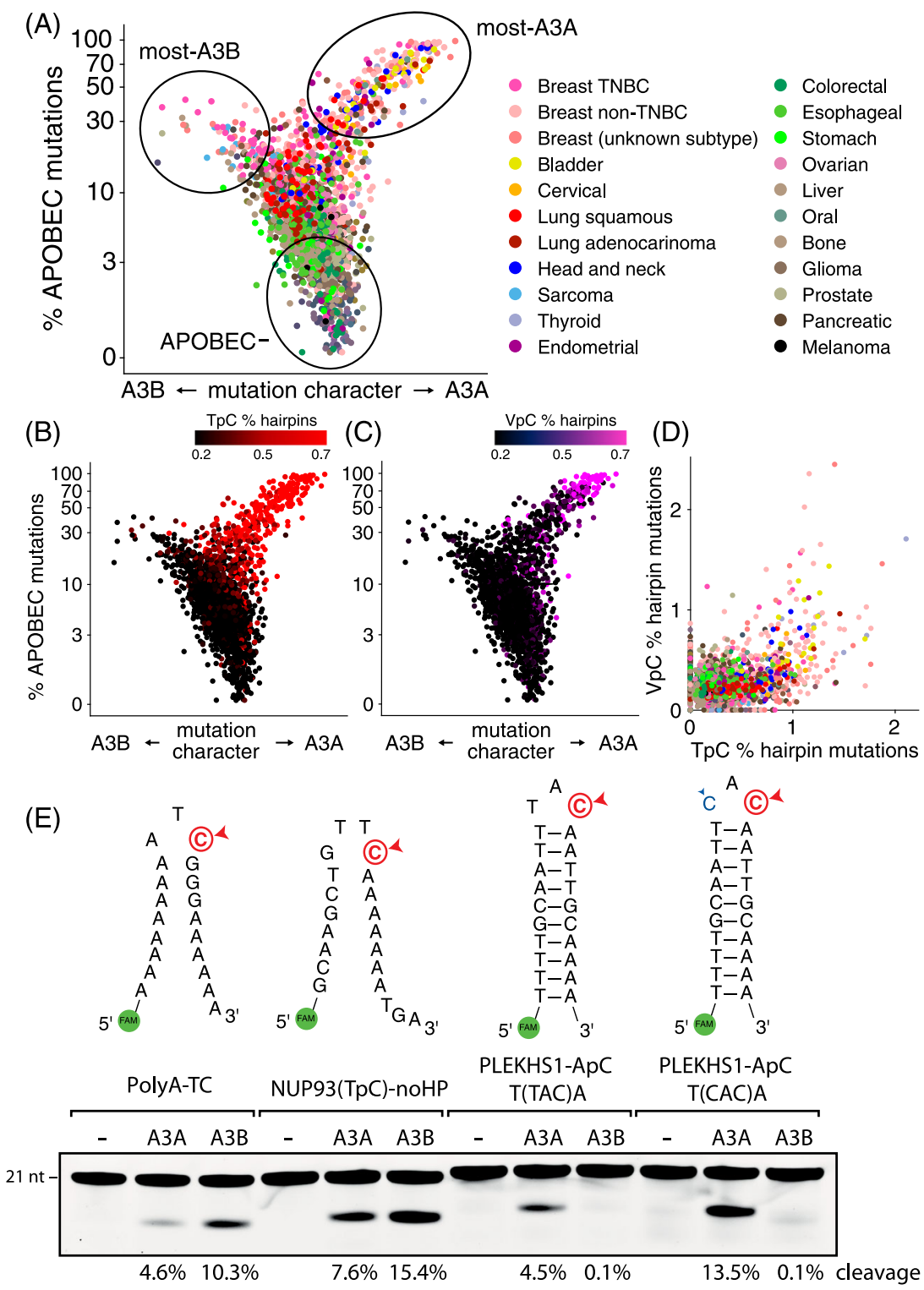

Fig. 3 Hairpin mutations at VpC sites and TpC sites are caused by APOBEC3A not APOBEC3B. A A set of tumors sequenced by WGS was stratified by two parameters: the $y$-axis separates APOBEC - tumors (bottom) from APOBEC + tumors (top), and the $x$-axis separates tumors with high A3B character (left) from those with high A3A character (right). Points are colored by tumor type (legend at right). B When tumors are colored by the fraction of APOBEC mutations occurring in TpC hairpins, it is clear that tumors enriched for TpC hairpin mutations (red) tend to be A3A-dominated APOBEC+ samples. C When tumors are colored by the fraction of APOBEC mutations occurring in $V p C$ hairpins, the same pattern is evident: tumors with the highest levels of $\mathrm{VpC}$ hairpin mutations (magenta) tend to be A3A-dominated tumors. D TpC and VpC hairpin mutations are tightly associated: tumors enriched for one are enriched for both, suggesting that $A 3 A$ is the joint cause of both. $\mathbf{E}$ Comparison of $A 3 A$ and $A 3 B$ activity on DNA oligos representing nonhairpin $T p C$ sites and hairpin $\mathrm{VpC}$ sites. A3A activity is seen on all substrates, but $A 3 B$ activity is not observed at $V p C$ sites. Source data are provided as a Source Data file.

reported $^{20}$ ), or for $\mathrm{VpC}$ hairpins. Finally, we observed that tumors showing high enrichments of TpC hairpin mutations are exactly the same tumors showing high enrichments of $\mathrm{VpC}$ hairpin mutations (Fig. 3D), confirming that the two classes of hairpin mutations are tightly associated, and supporting $\mathrm{A} 3 \mathrm{~A}$ as the single cause of both.

Next, to confirm that $\mathrm{A} 3 \mathrm{~A}$, and not $\mathrm{A} 3 \mathrm{~B}$, generates mutations at $\mathrm{VpC}$ hairpin sites, we compared the in vitro activity of $\mathrm{A} 3 \mathrm{~A}$ and $\mathrm{A} 3 \mathrm{~B}$ on a panel of DNA oligonucleotides. We used nonhairpin $\mathrm{TpC}$ substrates to determine the amounts of $\mathrm{A} 3 \mathrm{~A}$ and $\mathrm{A} 3 \mathrm{~B}$ showing comparable levels of APOBEC activity (Fig. 3E, left two substrates). When these amounts of $\mathrm{A} 3 \mathrm{~A}$ and $\mathrm{A} 3 \mathrm{~B}$ were tested on hairpin $\mathrm{VpC}$ substrates, only A3A but not A3B showed activity (Fig. 3E, right two substrates). We also showed the same pattern using endogenous APOBEC activity. The human pharyngeal squamous carcinoma cell line BICR6 expresses low levels of $\mathrm{A} 3 \mathrm{~A}$ at baseline and showed low activity on a $\mathrm{VpC}$ hairpin substrate (Supplementary Fig. 5A, B). However, stimulating the cells with gemcitabine and interferon alpha (GEM/IFNa) dramatically increased $\mathrm{A} 3 \mathrm{~A}$ protein amounts and increased cleavage of $\mathrm{VpC}$ hairpin substrates by up to tenfold (Supplentary Fig. $5 \mathrm{~A}-\mathrm{D})$. The effect was eliminated by co-administration of a small interfering RNA specifically against A3A (siA3A), returning cleavage levels to baseline. These results validate our finding that $\mathrm{A} 3 \mathrm{~A}$ is responsible for the observed activity at $\mathrm{VpC}$ hairpin sites. In contrast, the high levels of endogenous A3B in BICR6 cells 
(A)
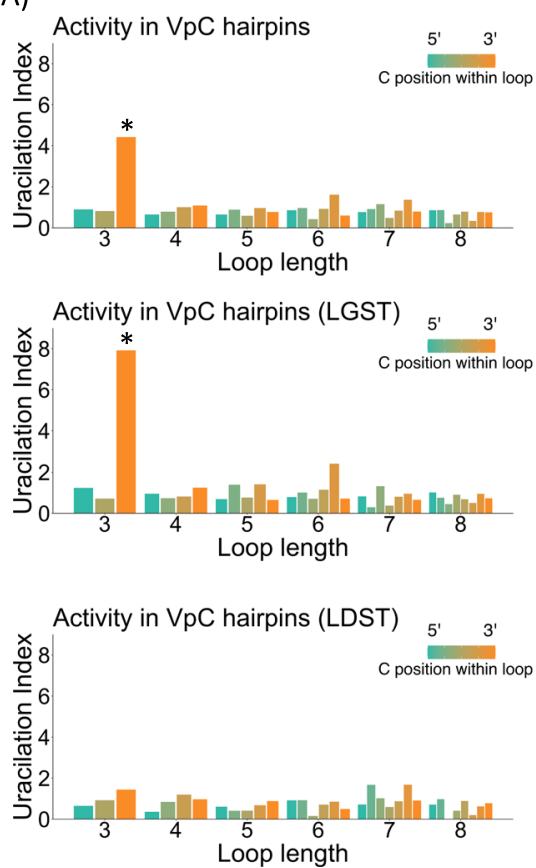

(B)
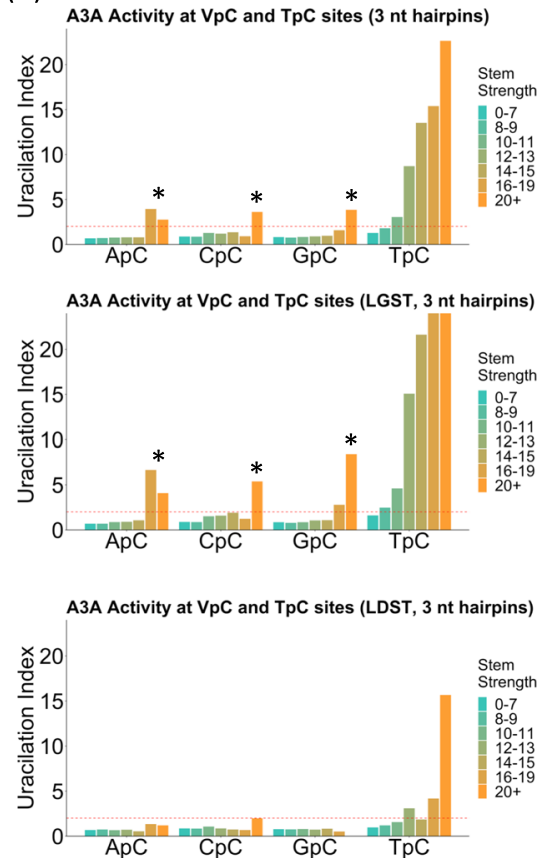

Fig. 4 APOBEC3A can deaminate $\mathbf{V p C}$ sites in bacteria. A Relative APOBEC3A activity, measured as Uracilation Index (UI), at cytosines in $\mathrm{VpC}$ sequences in predicted hairpin loops in the genome of $E$. coli. Increased activity is observed at cytosines in NVC triloops (asterisks), but only on the laggingstrand template (LGST). B Comparison of relative APOBEC3A activity in $\mathrm{VpC}$ sequences. Increased activity is seen at $\mathrm{VpC}$ sites in the loops of strongly paired hairpins (asterisks). Activity at hairpin $\mathrm{VpC}$ sites is several times stronger than at non-hairpin $\mathrm{TpC}$ sites. Activity at hairpin TpC sites is much higher than comparable $V p C$ sites. Source data are provided as a Source Data file.

showed no activity at $\mathrm{VpC}$ hairpin sites, but strong activity at non-hairpin TpC sites that was abrogated by treatment with an siRNA against A3B (Supplementary Fig. 5E-G). We confirmed this result using the human osteosarcoma cell line U2OS, which expresses no detectable A3A and high levels of A3B (Supplementary Fig. $5 \mathrm{H}$ ). At a normalized level of $\mathrm{A} 3 \mathrm{~B}$, cell extracts from U2OS and BICR6 cells showed similar activity at non-hairpin TpC sites, whereas U2OS cell extract showed no activity at VpC hairpin sites (Supplementary Fig. 5H, I).

Human APOBEC3A displays activity at $\mathrm{VpC}$ sites in the $E$. coli genome. Having observed $\mathrm{A} 3 \mathrm{~A}$ activity at non-canonical $\mathrm{VpC}$ sites in DNA hairpins in vitro and mutations at $\mathrm{VpC}$ sites in hairpin-forming sequences in tumors, we next investigated an in vivo laboratory model of A3A activity: bacterial cells expressing the human A3A enzyme. We described previously a method to enrich and sequence DNA fragments containing uracils (UPDseq). We applied this method to A3A-expressing E. coli cells ${ }^{21}$. Uracils created by $\mathrm{A} 3 \mathrm{~A}$ at various genomic positions were quantified by computing the uracilation index (UI) which is a quantitative measure of the frequency at which any cytosine in the genome is converted to uracil by A3A. This analysis showed that the UI at TpC's within predicted hairpin loops increased with stem strength and was highest for triloops with the target cytosine at the $3^{\prime}$ side of the loop (Supplementary Fig. 6A), exactly matching the patterns we reported previously for A3A activity in synthetic oligos and in human tumors. When these analyses were extended to $\mathrm{VpC}$ sequences in the $E$. coli genome, the highest UI values were found for $\mathrm{VpC}$ sites within hairpin loops with highest stem strengths and in which the cytosine was situated at the $3^{\prime}$ side of a triloop. $\mathrm{VpC}$ sites in strong hairpins showed four-times as much uracilation compared to non-hairpin $\mathrm{VpC}$ sites (Fig. 4A) and this effect was seen for all four possible $5^{\prime}$ nucleotides (Fig. 4B). This effect was not seen when analyzing cells expressing a catalytically inactive A3A point mutant or containing the empty vector (Supplementary Fig. 6B). This shows that while the magnitude of the UI is much greater for $\mathrm{TpC}$ sites in 3-nt hairpin loops than $\mathrm{VpC}$ sites even for strong hairpins (Fig. 4A, B, upper panels), A3A prefers cytosines in both these groups of sites over sites in larger loops. As noted previously ${ }^{21}$, the hairpin effect at TpC's was stronger for cytosines located on the LGST during replication than the LDST (Fig. 4B, lower panels). Expanding the strand asymmetry analysis to $\mathrm{VpC}$ sequences, we found that this was also true for $\mathrm{ApC}, \mathrm{CpC}$, and $\mathrm{GpC}$ sites, increasing to eightfold for cytosines at the $3^{\prime}$ side of triloops (Fig. 4A, B, lower panels). Together, these results confirm that $\mathrm{A} 3 \mathrm{~A}$ can show activity at sites previously considered not to be substrates for A3A activity, namely $\mathrm{VpC}$ sequences presented in hairpin loops. We see this $\mathrm{VpC}$ hairpin signature as completing an extended APOBEC $3 \mathrm{~A}$ signature and provide a new software tool ApoHP for quantifying hairpins and hairpin mutations.

Driver and passenger mutations at $\mathrm{VpC}$ sites in APOBEC+ tumors. If $\mathrm{VpC}$ sites in DNA hairpins are indeed substrates for $\mathrm{A} 3 \mathrm{~A}$ in tumors, then one would expect to see recurrent mutations at these sites in APOBEC+ tumors. We surveyed the top 100 most frequently mutated C:G basepairs, including both TpC and $\mathrm{VpC}$ sites, in our APOBEC+ cohort (Fig. 5). As previously noted, many of the top recurrent $\mathrm{TpC}$ mutations are not in known driver genes, but are in optimal hairpins. These $\mathrm{TpC}$ sites are likely hotspots of passenger mutations due to their high substrate optimality for $\mathrm{A} 3 \mathrm{~A}$. We also observe several $\mathrm{VpC}$ mutation hotspots in genes not known to confer a fitness advantage, at sites predicted to be highly optimal A3A substrates due to the favorable positioning of the cytosine at the $3^{\prime}$ end of a short loop closed by a strongly paired stem. For example, there are frequently mutated ApC hairpin sites in ADGRG6 (also called GPR126) and PLEKHS1. These hotspots are likely favored targets of A3A, easy 


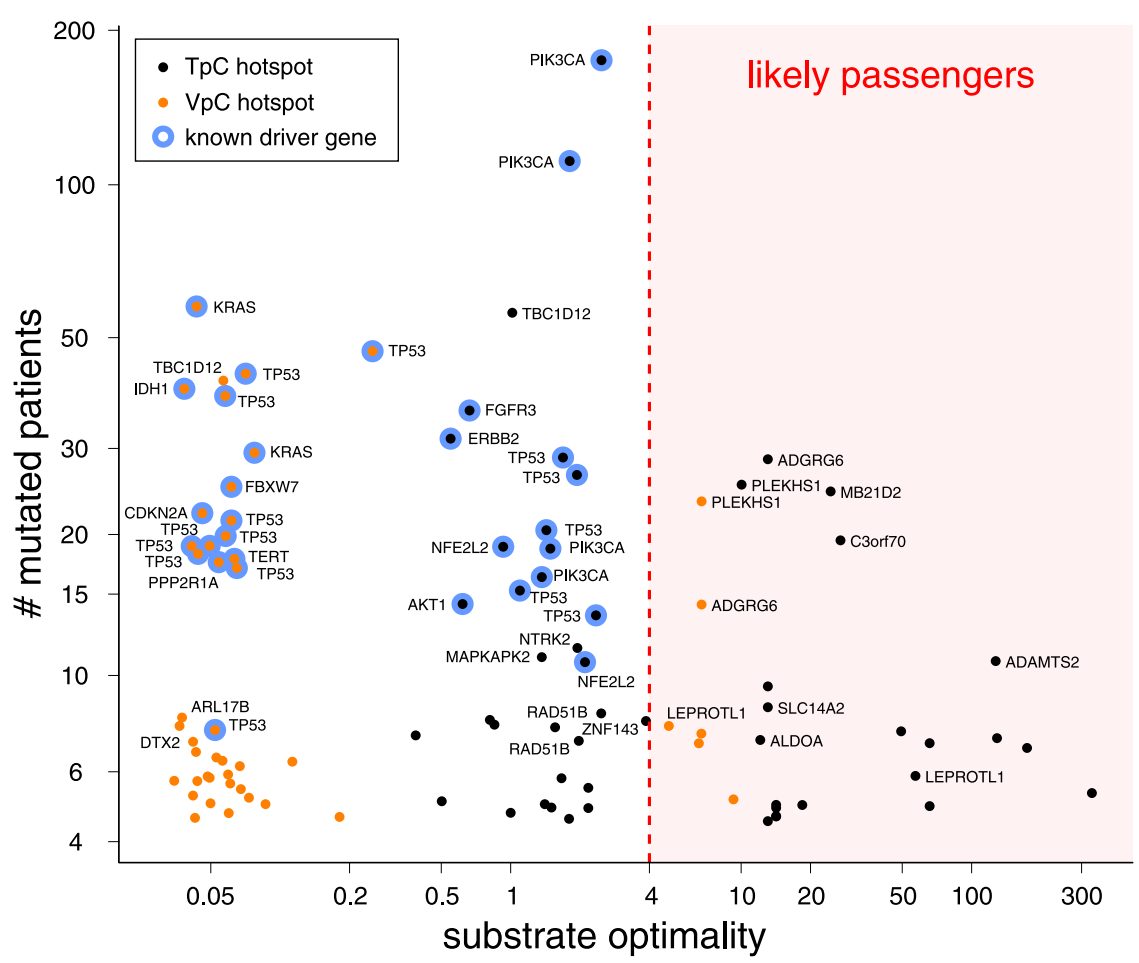

Fig. 5 VpC passenger hotspots. The top 100 mutation hotspots in a set of APOBEC+ tumors. The $x$-axis represents substrate optimality, the expected mutation frequency relative to the baseline mutation rate at all linear $\mathrm{TpC}$ sites. The $y$-axis represents the number of patients in this cohort carrying a mutation at that site. Hotspots at optimal hairpin substrates for APOBEC3A $(x \geq 4)$ are likely passenger hotspots, whereas hotspots at ordinary sites $(x<4)$ are known or likely drivers. VpC hotspots in PLEKHS1 and ADGRG6 (also called GPR126) can now be understood to be optimal APOBEC3A hotspots despite their non-canonical primary sequence. Source data are provided as a Source Data file.

to mutate due to their optimal secondary structure. Additional examples of recurrently mutated $\mathrm{VpC}$ hairpins are shown in Supplementary Fig. 7. These hotspots illustrate that the substrate preferences of $\mathrm{A} 3 \mathrm{~A}$ in vivo are broader than initially thought, and that many of the most recurrently mutated loci in APOBEC+ tumors can be properly explained as passenger hotspot mutations driven by the preference of $\mathrm{A} 3 \mathrm{~A}$ for hairpins.

In contrast to the recurrent $\mathrm{VpC}$ mutations in optimal hairpins, many other recurrent $\mathrm{VpC}$ mutations are at nonhairpin sites in known driver genes like KRAS and TP53. These sites have low predicted substrate optimality relative to the background linear-substrate $\mathrm{TpC}$ mutation rate. They are ordinary sites without the special structural properties that make for an optimal A3A substrate.

The extended APOBEC3A mutation signature helps explain the genomic Twin Paradox. Our broadened definition of A3A substrate preferences sheds light on the genomic Twin Paradox, revealing that these twin hotspots are likely entirely APOBECcaused. Specifically, our in vivo tumor statistics suggest a model in which the occurrence of the first mutation, followed by DNA replication and cell division, leads to propagation of the mutation to the opposite strand, resulting in a reduction of that strand's fitness for $\mathrm{A} 3 \mathrm{~A}$, and lowering the probability of incurring the second mutation (Fig. 6A, B). In short, the first mutation inhibits occurrence of the second. This model is supported by our in vitro biochemical experiments using a series of synthetic DNA oligos modeling the PLEKHS1 twin hotspot (Fig. 6C). We find that the $\mathrm{VpC}$ site in the last position of the loop of the twin hotspot hairpins is most mutable with a guanine in the first position in the loop, i.e., in the reference configuration of the loop. Importantly, mutating the TpC nucleotide in the first position of the loop through $\mathrm{A} 3 \mathrm{~A}$ mutagenesis drastically reduces the mutability of the neighboring $\mathrm{VpC}$ site. Thus, these in vitro assays recapitulate the A3A-mediated mutagenesis at a twin hotspot, confirming that both the $\mathrm{TpC}$ and $\mathrm{VpC}$ sites are efficient substrates of A3A. Furthermore, when the mutation at the TpC is made permanent through DNA replication, it suppresses the occurrence of the mutation at the $\mathrm{VpC}$ site, explaining why these mutation pairs are seldom, perhaps never, observed in cis at the twin hotspot, and resolving the genomic Twin Paradox: these are simply pairs of closely situated A3A passenger hotspots.

\section{Discussion}

Mutations caused by APOBEC enzymes are one of the most prevalent mutation signatures in cancer ${ }^{26}$ and have been studied intensively in recent years ${ }^{3}$. The signature has been known by its various separate aspects, such as its knack for generating strandcoordinated mutation clusters called kataegis $^{17,26,33,34}$, its unusual tendency to generate mutations in gene-rich, early replicating, active chromatin ${ }^{35}$, its strand-asymmetric impact on the replicative LGST ${ }^{27-30}$, and its proclivity to mutate DNA and RNA hairpin loops ${ }^{18-20,36}$; but most of all, the APOBEC signature has been commonly understood as restricted to $\mathrm{TpC}$ sites ${ }^{13-15}$. Through our combined biochemical and bioinformatic analyses, we have shown that this understanding is incomplete: hairpin DNA sites can be optimal substrates for A3A even if they are not TpC sites. This reveals an extended APOBEC mutation signature that is broader than previously believed. In particular, many mutations previously thought due to other signatures, such as the Aging signature, may actually be due to APOBEC.

The activity of $\mathrm{A} 3 \mathrm{~A}$ at cytosines other than $\mathrm{TpC}$ sites has escaped notice in most analyses of the APOBEC mutational signature because enzymatic activity at the vast majority of $\mathrm{VpC}$ sites is very weak, as most sites in the genome are not able to form a hairpin. However, when we focus our attention on hairpin sites, 
(A)

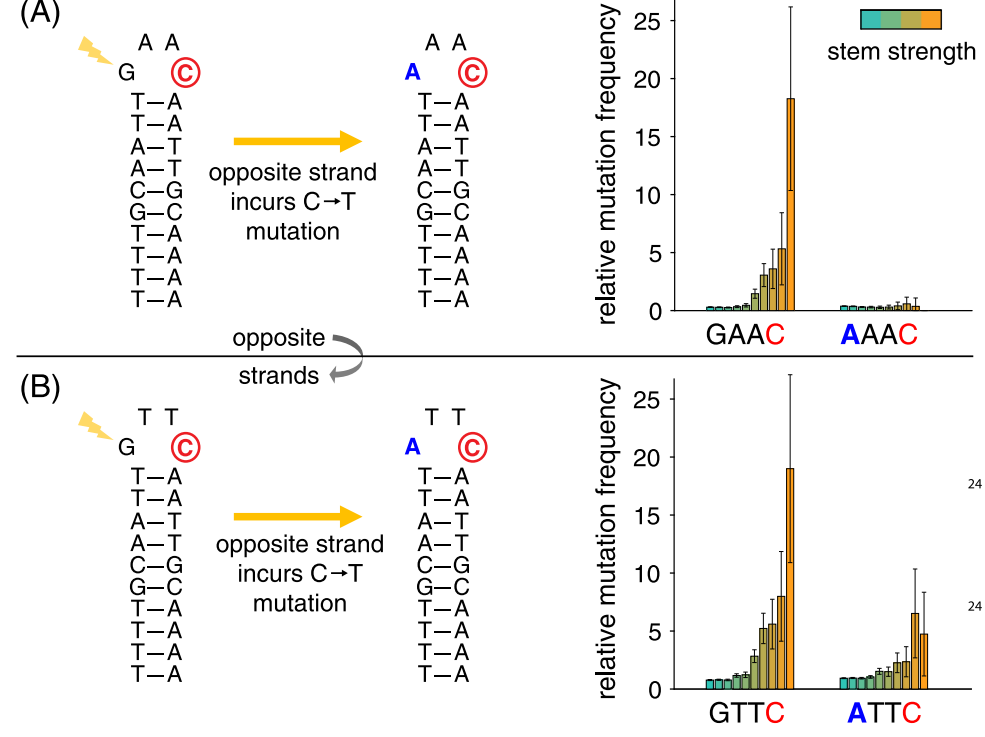

(C)
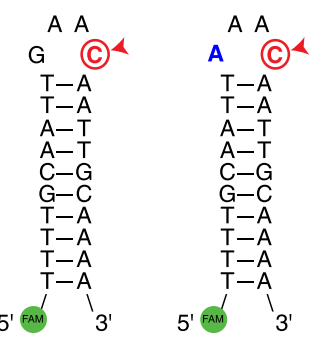

PLEKHS1-ApC T(GAAC)A

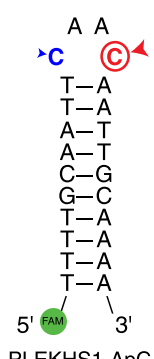

PLEKHS1-ApC

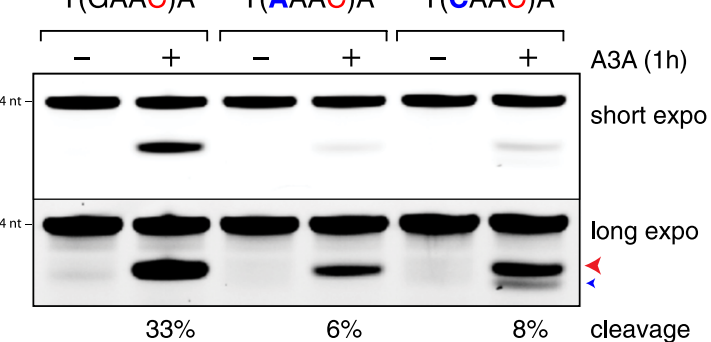

Fig. 6 Twin mutation hotspot combining optimal TpC and VpC sites. A twin mutation hotspot detected in the gene PLEKHS1 contains an optimal hairpin substrate on each strand of the DNA. A On one DNA strand, the hairpin contains the sequence GAAC, and the $\mathrm{C}$ in last position is an optimal $\mathrm{VpC}$ substrate for A3A. B On the other DNA strand, the hairpin contains the sequence GTTC, and the C in last position is an optimal TpC substrate for A3A. However, when A3A generates a mutation at either strand's site, and the cell goes through DNA replication, then the mutation becomes propagated to the complementary strand, in the first $\left(5^{\prime}\right)$ position of the loop. This mutation causes the local sequence context to no longer be optimal, leading to a decrease in the observed mutation frequency in tumors (bar plots). Error bars represent $95 \%$ confidence intervals. C In vitro APOBEC $3 \mathrm{~A}$ assay confirms activity at GAAC hairpin loop sequence. Changing to AAAC or CAAC reduces the substrate optimality by corrupting the optimal GAAC sequence. Source data are provided as a Source Data file.

we are able to clearly see the activity of $\mathrm{A} 3 \mathrm{~A}$ at $\mathrm{VpC}$ sites. The preference for a $\mathrm{T}$ (and/or a hairpin turn) at the $5^{\prime}$-position may be due to the conformation of the target cytosine at the end of the loop. It has been shown that thymines contribute the least to base stacking ${ }^{37,38}$ and poly-dT is the most flexible homopolymer ${ }^{39}$. This flexibility may underlie A3A's usual preference for a T preceding its substrate $\mathrm{C}$. A3A may target linear $\mathrm{TpC}$ substrates due to the preceding $\mathrm{T}$ being able to move out of the way as $\mathrm{A} 3 \mathrm{~A}$ flips the neighboring cytosine into its active site. We suggest that the enforced sharp turn of a hairpin loop may prepay some of the entropic cost of this interaction (Fig. 7).

Our results in E. coli show that cytosines in hairpins are much more susceptible to deamination if they are on the LGST than the LDST, and this effect is strongest for cytosines at $3^{\prime}$ ends of triloops connected to stems with expected high stability. While the effect is seen with all C's, it is much stronger for TpC's than $\mathrm{VpC}$ 's. This is in agreement with our previous reports ${ }^{27,28}$ that APOBEC mutations are enriched on the LGST and this is true of mutations in both $\mathrm{TpC}$ and $\mathrm{VpC}$ sequence contexts (Supplementary Fig. 4). Our data suggest that at the replication fork, RPA (or SSB in E. coli) is unable to bind ssDNA that can form strong hairpins. RPA wraps a long stretch $(\sim 30 \mathrm{nt})$ of ssDNA around itself without using a high energy cofactor such as ATP ${ }^{40,41}$. Consequently, (1) high stem strength is essential to prevent binding of RPA: if a hairpin with high stem strength forms on the LGST, RPA would be unable to break the base pairing within the stem and bind the DNA; and (2) cytosines within loops of such hairpins are much more susceptible to deamination by A3A than those in random-coil ssDNA (Supplementary Fig. 8).

The greater susceptibility of cytosines in short loops to deamination by A3A probably reflects both (1) an intrinsic structural preference of $\mathrm{A} 3 \mathrm{~A}$, as the sharp bending required to fit the substrate DNA into the active site of the enzyme ${ }^{8,42}$ is already present in short loops; as well as (2) a tendency of bases in short loops to flip out because of the strain and lack of base pairing. Previous work has posited that a preceding thymine may also play a role in stabilizing the aromatic residues in the hydrophobic binding pocket of $\mathrm{A} 3 \mathrm{~A}$, implying that $\mathrm{A} 3 \mathrm{~A}$ has inherent sequence specificity in its binding capability ${ }^{8}$. However, cytosines in short loops (especially triloops) fit so well in the active site of A3A that the enzyme, even without hydrogen bonds to the $5^{\prime}-\mathrm{T}$, can bind and deaminate them. This suggests that the catalytic advantage conferred by hairpin loops is an additive effect contributing to A3A substrate preferences, on top of underlying inherent primary sequence preferences. It is also possible that, as a member of an ancient family of zinc-dependent viral-defense enzymes, A3A evolved specifically for its ability to mutate hairpin loops, to aid in the recognition and defense against ssDNA viruses, which are known to adopt intricately folded structures ${ }^{43}$.

The observation of recurrent $\mathrm{VpC}$ mutations at poor $\mathrm{A} 3 \mathrm{~A}$ substrate sites in known driver genes in APOBEC+ tumors suggests that A3A may contribute to tumor fitness, although we cannot exclude the possibility that these mutations are generated by A3Aindependent mechanisms. Recurrent $\mathrm{VpC}$ mutations at non-hairpin sites are found not only in known drivers, but also in genes not known to be drivers. These genes carrying recurrent $\mathrm{VpC}$ mutations at poor $\mathrm{A} 3 \mathrm{~A}$ substrate sites are possible novel drivers.

Understanding the factors that drive mutagenesis enables identification of driver mutations, which is crucial for the elucidation of the biological mechanisms of diseases and the efficient pursuit of novel therapeutics. Driver hotspots are specific genomic loci where mutations arise infrequently and stochastically, but are selected for and clonally expanded due to the strong fitness advantage they confer. In contrast, passenger hotspots ${ }^{44}$ are specific genomic loci where mutations occur frequently and predictably, but do not confer any fitness advantage. Distinguishing drivers from passengers is still not a fully solved problem in cancer genomics. We showed previously that $\mathrm{A} 3 \mathrm{~A}$ recurrently targets specific hairpin loci in the genome, leading to mutation hotspots in the absence of selective benefit. Our discovery that A3A can efficiently deaminate cytosines lacking a $5^{\prime}-\mathrm{T}$ if they occur in optimal hairpin structures allows us to explain the twin hotspot 


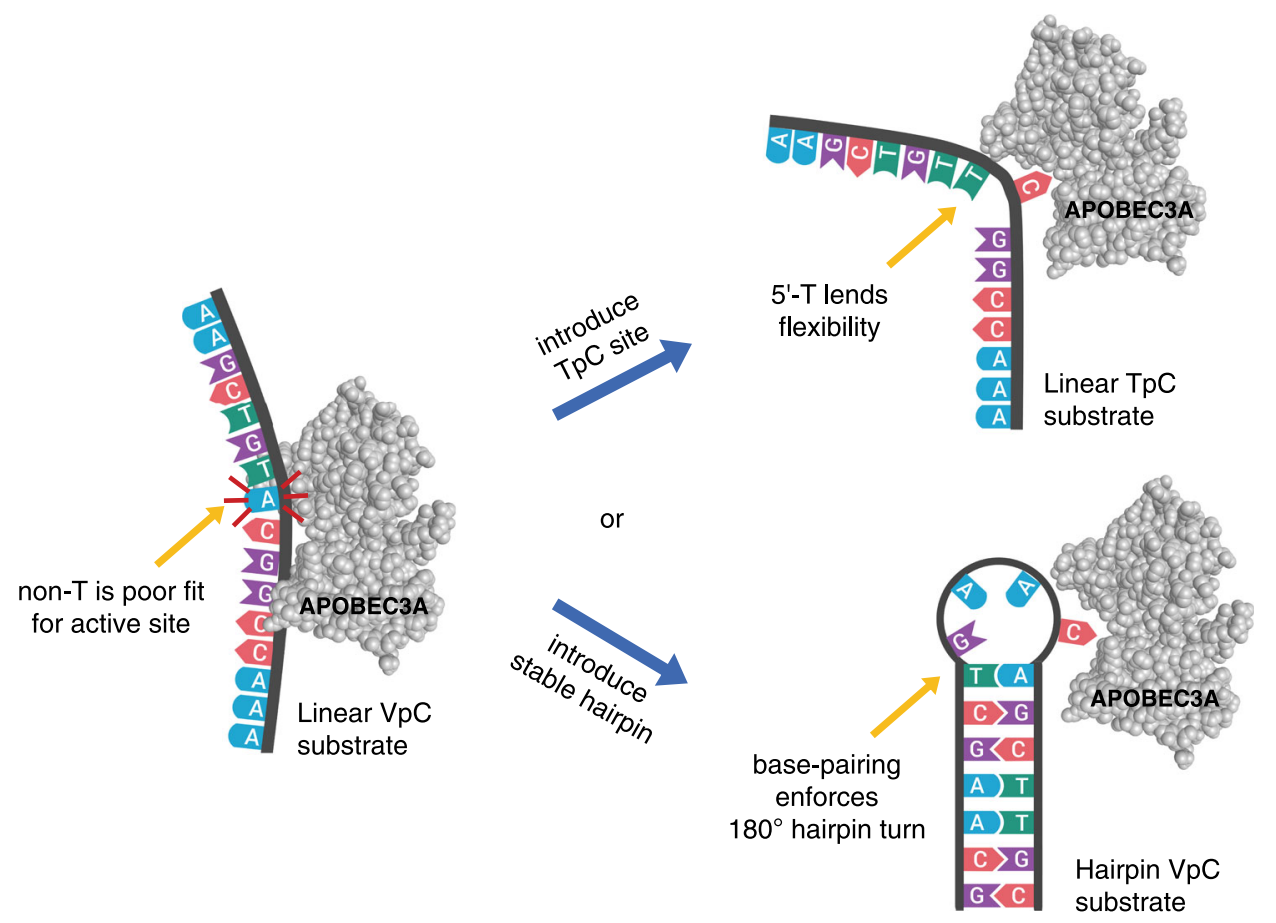

Fig. 7 Determinants of APOBEC3A substrate optimality. Linear (non-hairpin) DNA substrate containing a $V p C$ sequence has both non-optimal sequence and non-optimal structure, causing it to be a poor substrate for APOBEC3A. However, improving either the primary sequence (top path) or secondary structure (bottom path) improves the overall substrate optimality and enables APOBEC $3 \mathrm{~A}$ activity. Changing the $\mathrm{VpC}$ sequence to TpC enables productive contacts between the $T$ and the enzyme, and may also increase flexibility at the precise point where the DNA needs to bend to fit into the A3A active site. Keeping the $\mathrm{VpC}$ sequence but changing the structure to a hairpin prepays the entropic cost of bending the DNA at the $5^{\prime}$ site, enforcing a 180 -degree hairpin turn. (Figure created with BioRender.com).

loci discussed above: these are a combination of two optimal substrates for A3A. These twin hotspots are fascinating bipartite mimics, pairs of sites that perfectly masquerade as two-hit tumor suppressors. Indeed, twin hotspot mutations in the gene ADGRG6 (also called GPR126) were recently proposed ${ }^{25}$ as a potential novel driver of angiogenesis in bladder cancer. Our results show they are better understood as an exotic dually optimal mutagenic substrate. Other mutational processes are likely to recurrently target specific sequence/structure combinations, and understanding the causes of these passenger hotspots will help to separate true driver mutations from false-positive pseudo-drivers.

Separating true driver events from passenger mutation hotspots is crucial for effective analysis and clinical intervention in the diagnosis and treatment of cancer, particularly with the advent of targeted therapies. Knowing which mutations confer real fitness advantages to tumors and are thus worth investigating and targeting is an essential step when analyzing cancer genomic data. Furthermore, incorporating structural features into mutation signature analysis leads to a fundamentally expanded definition of an important endogenous mutational process. We anticipate that other forms of DNA structure, e.g., short cytosinecontaining bulges or loops in structures like G-quadruplexes ${ }^{45,46}$ may also influence the activity of APOBEC and other mutational processes.

\section{Methods}

Plasmids. APOBEC3A and APOBEC3B cDNA were synthesized by GenScript with a beta-globin intron and a Flag tag at $\mathrm{C}$-terminus. The plasmid expressing APOBEC3A-Flag, and APOBEC3B-Flag were generated by inserting the cDNA into pDEST53 vectors using the Gateway Cloning System (Thermo Fisher Scientific).

Cell culture. HEK-293T cells were maintained in DMEM supplemented with $10 \%$ FBS and 1\% penicillin/streptomycin. HEK-293T cells were transfected with
pDEST53-APOBEC3A-Flag or pDEST53-APOBEC3B-Flag using Lipofectamine 2000 (Thermo Fisher Scientific). BICR6 was maintained in DMEM/F12 GlutaMAX ${ }^{\prime \prime}-\mathrm{I}$ supplemented with $10 \% \mathrm{FBS}$ and $1 \%$ penicillin/streptomycin. The cell lines above were purchased from either ATCC or Millipore-Sigma.

Chemicals. Gemcitabine was purchased from Selleckchem and dissolved in DMSO. Purified human Interferon- $\alpha \mathrm{A} / \mathrm{D}$ was purchased from Millipore-Sigma.

Cell extracts. HEK-293T cells or HEK-293T cells expressing A3A or A3B were lysed in $25 \mathrm{mM}$ HEPES ( $\mathrm{pH} 7.9$ ), $10 \%$ glycerol, $150 \mathrm{mM} \mathrm{NaCl}, 0.5 \%$ Triton X-100, $1 \mathrm{mM}$ EDTA, $1 \mathrm{mM} \mathrm{MgCl}$, and $1 \mathrm{mM} \mathrm{ZnCl} 2$ and protease inhibitors. Cell lysates were sonicated and centrifuged $10 \mathrm{~min}$ at $16,000 \times g$ at $4{ }^{\circ} \mathrm{C}$. Then, RNase $\mathrm{A}(0.2$ $\mu \mathrm{g} / \mathrm{ml}$ ) was added for $30 \mathrm{~min}$ at $4^{\circ} \mathrm{C}$ and cell lysates were centrifuged $10 \mathrm{~min}$ at $16,000 \times g$ at $4{ }^{\circ} \mathrm{C}$. Protein concentration of the supernatant was determined by Bradford assay (Bio-Rad).

DNA deaminase activity assay. The deamination assays were performed as previously described ${ }^{33,47,48}$. Reactions $(50 \mu \mathrm{l})$ containing $8 \mu \mathrm{l}$ of a normalized amount of cell extracts (expressing A3A or A3B) were incubated at $37^{\circ} \mathrm{C}$ during $1 \mathrm{~h}$ in a reaction buffer $(42 \mu \mathrm{l})$ containing a DNA oligonucleotide (20 pmol of DNA oligonucleotide, $50 \mathrm{mM}$ Tris (pH 7.4), 1.5 units of uracil DNA glycosylase (NEB), and $10 \mathrm{mM}$ EDTA). Then, $100 \mathrm{nM}$ of $\mathrm{NaOH}$ was added to the reaction following by $40 \mathrm{~min}$ incubation at $95^{\circ} \mathrm{C}$. Finally, $50 \mu \mathrm{l}$ of formamide was added to the reaction $\left(50 \%\right.$ final) and the reaction was incubated at $95^{\circ} \mathrm{C}$ for $10 \mathrm{~min}$ following by $5 \mathrm{~min}$ at $4{ }^{\circ} \mathrm{C}$. DNA cleavage was monitored on a $20 \%$ denaturing acrylamide gel (8 M urea, $1 \mathrm{X} \mathrm{TAE}$ buffer) and run at $65^{\circ} \mathrm{C}$ for $80 \mathrm{~min}$ at $150 \mathrm{~V}$. DNA cleavage of PLEKHS1- ApC-T(CAC)A oligonucleotide was monitored on a $24.5 \%$ denaturing acrylamide gel ( $8 \mathrm{M}$ urea, $1 \mathrm{X}$ TAE buffer) and run at $65^{\circ} \mathrm{C}$ for $4 \mathrm{~h}$ at $150 \mathrm{~V}$. DNA oligonucleotide probes were synthetized by Thermo Fisher Scientific. The sequences of DNA oligonucleotide probes used in this study are listed in Supplementary Table 1 .

Bioinformatic analyses. The human genome (build hg19) was scanned for potential hairpin-forming sequences using the survey hairpins function of the ApoHP tool [http://github.com/alangenb/ApoHP], which implements a version of the algorithm described in previous work ${ }^{20}$. Briefly, the genome was scanned for sequences of the form S-L-S', where the sequences $\mathrm{S}$ and $\mathrm{S}^{\prime}$ are reversecomplementary with a sequence L (ranging from 3 to 11 nucleotides) intervening between them. Sequences such as these have the potential to form stem-loop, or 
"hairpin" structures in DNA that is transiently single-stranded. For each position $\mathrm{p}$ in the genome, flanking sequences $S$ and $S^{\prime}$ were sought such that position $p$ would be in the intervening loop sequence L. Stem strength was defined as the number of A:T basepairs plus $3 \times$ the number of G:C basepairs, an approximation of empirically measured nearest-neighbor stacking energies ${ }^{49}$. In cases where multiple alternative pairings were possible, the stem with the strongest pairing was chosen, using shortest loop size as a tie-breaker. The output of this procedure was to assign to each genomic position a set of parameters describing its hairpin characteristics: stem strength, loop length (in nucleotides), and position of the mutation-site cytosine within the loop (ranging from 1 to loop length). This allows genomic positions to be categorized into equivalence classes for investigating the influence of hairpin characteristics on relative mutation frequency, which we describe below.

Mutation calls from whole-genome sequencing (WGS) were obtained from TCGA and the International Cancer Genome Consortium Pan-Cancer Analysis of Whole Genomes project, and other projects ${ }^{1,22}$, as described below in the "Data availability" section. We restricted our analysis to somatic single-nucleotide variants (SSNVs) and excluded patients with fewer than 500 SSNVs in the genome, yielding a final WGS dataset comprising 2800 unique patients spanning 35 tumor types. Mutations were analyzed by the analyze mutations function of ApoHP. Mutation signatures were analyzed by NMF, using $k=8$, revealing a set of signatures corresponding to known mutational processes, including APOBEC enzyme activity. Each patient was characterized by frac_apobec, the fraction of its mutations assigned to the APOBEC signature. Cohorts of APOBEC+ patients were defined by imposing a threshold on frac_apobec. Our initial analyses used a set of 110 patients with frac_apobec $\geq 50 \%$. We repeated some analyses using a cutoff of $10 \%$ or $90 \%$, with similar results. To minimize the influence of potentially overlapping hypermutation processes such as MSI, Smoking, UV, POLE, or ESO, we excluded "MSUPE+" patients, those with at least $10 \%$ of their mutations assigned by NMF to one of these five other hypermutation processes.

To measure the joint influence of hairpin-forming potential and local sequence context on the relative mutation frequency of APOBEC signature mutations, we binned genomic cytosines into classes of equivalent cytosines that share hairpin characteristics and local sequence features. For each class of genomic cytosines, we counted the number of sites $(N)$ in the genome that belong to this class, as well as the number of mutations $(n)$ observed in the APOBEC + cohort being analyzed. We then calculated the ratio $(n / N)$, which represents the number of mutations per sites of this class. For classes of cytosines that have few sites in the genome and/or few observed mutations, the uncertainty on this estimated relative mutation frequency becomes large. Therefore, we estimated a $95 \%$ confidence interval on the ratio, by first approximating the standard deviation (SD) of the ratio by propagating an assumed square-root uncertainty in the integer counts, using the formula $\mathrm{SD}=(n / N) \times\left[\left(n^{1 / 2} / n\right)^{2}+\left(N^{1 / 2} / N\right)^{2}\right]^{1 / 2}$, and then estimating the $95 \%$ confidence interval as $(n / N) \pm 1.96 \times \mathrm{SD}$. Finally, we normalized this ratio (and confidence interval) to a relative mutation frequency, by dividing the APOBEC+ cohort's baseline rate of mutations at all TCA trinucleotides in the genome (the most favored site for APOBEC mutations). The bar plots in Figs. 2A, B and 6A, B and Supplementary Fig. 2A, B show this estimated relative mutation frequency, with the estimated $95 \%$ confidence interval shown as error bars. This analysis is part of the analyze mutations function of ApoHP.

Quantitative modeling to predict relrate_exp for any given cytosine base in the genome was performed by the analyze_mutations function of ApoHP, and leveraged the observation that mutation rate in hairpin loops increases as an exponential function of stem strength ${ }^{20}$. We fit a global variable $m_{\mathrm{hp}}=$ the multiplicative increase in mutation frequency with each additional unit of stem strength. For each type of hairpin, defined by the loop length, loop position, and local sequence context, we fit a local parameter $\mathrm{ss}_{\mathrm{o}}=$ the stem strength necessary to reach double the baseline non-hairpin mutation rate for that type of hairpin. For some hairpin types (e.g., with the $\mathrm{C}$ at the wrong side of the loop), mutation rate never rises above baseline, and $\mathrm{ss}_{\mathrm{o}}$ is high. For optimal hairpins, mutation rate rises very quickly with increasing stem strength, and $\mathrm{ss}_{\mathrm{o}}$ is low.

Uracil pull-down and sequencing of bacterial genomic DNA. Wild-type A3A, its E72A mutant, or the empty vector were introduced in an $u n g^{-} \mathrm{mug}^{-}$strain of $E$. coli, and uracil-containing genomic DNA fragments were pulled-down and sequenced (UPD-seq) in previously published work ${ }^{21}$. Briefly, the genomic DNA was isolated and uracils were excised using E. coli Ung enzyme, and the resulting abasic sites were labeled using a chemical, ssARP, that contains a biotin. These labeled fragments were separated from the non-uracil-containing fragments using streptavidin beads, chemically cleaved off the beads and subjected to NextGen sequencing. The sequencing reads are available at the NCBI SRA (short read archive) under BioProject ID: PRJNA448166 (https://www.ncbi.nlm.nih.gov/ bioproject/PRJNA448166/). The UI was defined as the fraction of the sequencing reads at a genomic reference cytosine that are called as thymine, multiplied by 1000. The potential hairpin loops in the E. coli genome were predicted using the same criteria used for the cancer genomes, and UI was calculated for predicted hairpin loops with different stem strengths, loop sizes, and loop sequences ${ }^{21}$.

Statistics and reproducibility. All western blots and DNA gels were repeated at least three times and representative images are shown in this paper.

\section{Data availability}

This study analyzed published data and did not generate any new sequencing data. Sequencing reads from the uracil pull-down experiments ${ }^{21}$ are available at the NCBI SRA (short read archive) under BioProject ID: PRJNA448166. Mutation calls from TCGA wholeexome sequencing (WXS) were obtained from the TCGA Unified Ensemble MC3 Call Set ${ }^{50}$ the public, open-access dataset of somatic mutation calls produced by the MC3 calling effort (Multi-Center Mutation Calling in Multiple Cancers), downloaded from the following link: [http://www.synapse.org/\#!Synapse:syn7214402/wiki/405297] (The results here are in whole or part based upon data generated by the TCGA Research Network: [http://cancergenome. nih.gov/] as outlined in the TCGA publications guidelines [http://cancergenome.nih.gov/ publications/publicationguidelines]). Following the filtering procedure that was used for the PanCanAtlas project, the MC3 dataset was filtered to include only PASS variants, which removes patients that were subjected to whole-genome amplification (WGA), as well as the acute myeloid leukemia (LAML) cohort. This yielded a final cohort of 9023 patients covering 32 tumor types. Mutation calls from whole-genome sequencing (WGS) from TCGA and other projects were obtained from the International Cancer Genome Consortium (ICGC) Pan-Cancer Analysis of Whole Genomes (PCAWG) project. Mutation calls were downloaded from the ICGC Portal (https://dcc.icgc.org/api/v1/download?fn=/PCAWG/ consensus_snv_indel/final_consensus_passonly.snv_mnv_indel.icgc.public.maf.gz and https://dcc.icgc.org/api/v1/download?fn=/PCAWG/consensus_snv_indel/ final_consensus_snv_indel_passonly_icgc.public.tgz). Note that controlled tier access credentials are required from the ICGC and TCGA projects as described on the ICGC PCAWG site [http://docs.icgc.org/pcawg/data/]. Additional WGS data were obtained from published projects ${ }^{1,22}$ from the following links: [ftp://ftp.sanger.ac.uk/pub/cancer/ AlexandrovEtAl/somatic_mutation_data] and [ftp://ftp.sanger.ac.uk/pub/cancer/Nik ZainalEtAl-560BreastGenomes/Caveman_560_20Nov14_clean.txt]. Identifying and removing duplicate patients, restricting to somatic single-nucleotide variants (SSNVs), and excluding patients with fewer than 500 SSNVs in the genome yielded a final WGS dataset comprising 2800 unique patients spanning 35 tumor types. Gene expression measurements from TCGA RNA-Seq were obtained from the Broad Institute GDAC website [http://gdac. broadinstitute.org/runs/stddata_2014_09_02/data]. Source data are provided with this paper.

\section{Code availability}

Source code and executable software tool ApoHP are available at http://github.com/ alangenb/ApoHP and have been archived via Zenodo ${ }^{51}$.

Received: 4 March 2020; Accepted: 17 February 2021; Published online: 11 March 2021

\section{References}

1. Alexandrov, L. B. et al. Signatures of mutational processes in human cancer. Nature 500, 415-421 (2013).

2. Lawrence, M. S. et al. Mutational heterogeneity in cancer and the search for new cancer-associated genes. Nature 499, 214-218 (2013).

3. Cortez, L. M. et al. APOBEC3A is a prominent cytidine deaminase in breast cancer. PLoS Genet 15, e1008545 (2019).

4. Petljak, M. et al. Characterizing mutational signatures in human cancer cell lines reveals episodic APOBEC mutagenesis. Cell 176, 1282-1294 e1220 (2019).

5. Venkatesan, S. et al. Perspective: APOBEC mutagenesis in drug resistance and immune escape in HIV and cancer evolution. Ann. Oncol. 29, 563-572 (2018).

6. Law, E. K. et al. APOBEC3A catalyzes mutation and drives carcinogenesis in vivo. J. Exp. Med. 217 https://doi.org/10.1084/jem.20200261 (2020).

7. Chen, $\mathrm{H}$. et al. APOBEC3A is a potent inhibitor of adeno-associated virus and retrotransposons. Curr. Biol. 16, 480-485 (2006).

8. Shi, K. et al. Structural basis for targeted DNA cytosine deamination and mutagenesis by APOBEC3A and APOBEC3B. Nat. Struct. Mol. Biol. 24, 131-139 (2017).

9. Bishop, K. N. et al. Cytidine deamination of retroviral DNA by diverse APOBEC proteins. Curr. Biol. 14, 1392-1396 (2004).

10. Henderson, S., Chakravarthy, A., Su, X., Boshoff, C. \& Fenton, T. R. APOBEC mediated cytosine deamination links PIK3CA helical domain mutations to human papillomavirus-driven tumor development. Cell Rep. 7, 1833-1841 (2014).

11. Henderson, S. \& Fenton, T. APOBEC3 genes: retroviral restriction factors to cancer drivers. Trends Mol. Med. 21, 274-284 (2015).

12. Roberts, S. A. et al. Clustered mutations in yeast and in human cancers can arise from damaged long single-strand DNA regions. Mol. Cell 46, 424-435 (2012).

13. Thielen, B. K. et al. Innate immune signaling induces high levels of TC-specific deaminase activity in primary monocyte-derived cells through expression of APOBEC3A isoforms. J. Biol. Chem. 285, 27753-27766 (2010). 
14. Martinez, T., Shapiro, M., Bhaduri-McIntosh, S. \& MacCarthy, T. Evolutionary effects of the AID/APOBEC family of mutagenic enzymes on human gamma-herpesviruses. Virus Evol. 5, vey040 (2019).

15. Suspene, R. et al. Self-cytoplasmic DNA upregulates the mutator enzyme APOBEC3A leading to chromosomal DNA damage. Nucleic Acids Res. 45, 3231-3241 (2017).

16. Georgakopoulos-Soares, I., Morganella, S., Jain, N., Hemberg, M. \& Nik-Zainal, S. Noncanonical secondary structures arising from non-B DNA motifs are determinants of mutagenesis. Genome Res. 28, 1264-1271 (2018).

17. Nik-Zainal, S. et al. Mutational processes molding the genomes of 21 breast cancers. Cell 149, 979-993 (2012).

18. Jalili, P. et al. Quantification of ongoing APOBEC3A activity in tumor cells by monitoring RNA editing at hotspots. Nat. Commun. 11, 2971 (2020).

19. Sharma, S. \& Baysal, B. E. Stem-loop structure preference for site-specific RNA editing by APOBEC3A and APOBEC3G. PeerJ 5, e4136 (2017).

20. Buisson, R. et al. Passenger hotspot mutations in cancer driven by APOBEC3A and mesoscale genomic features. Science 364 https://doi.org/ 10.1126/science.aaw2872 (2019).

21. Sakhtemani, R. et al. Genome-wide mapping of regions preferentially targeted by the human DNA-cytosine deaminase APOBEC3A using uracil-DNA pulldown and sequencing. J. Biol. Chem. 294, 15037-15051 (2019).

22. Nik-Zainal, S. et al. Landscape of somatic mutations in 560 breast cancer whole-genome sequences. Nature 534, 47-54 (2016).

23. Rheinbay, E. et al. Recurrent and functional regulatory mutations in breast cancer. Nature 547, 55-60 (2017).

24. Pignot, G. et al. PLEKHS1: a new molecular marker predicting risk of progression of non-muscle-invasive bladder cancer. Oncol. Lett. 18, 3471-3480 (2019).

25. $\mathrm{Wu}, \mathrm{S}$. et al. Whole-genome sequencing identifies ADGRG6 enhancer mutations and FRS2 duplications as angiogenesis-related drivers in bladder cancer. Nat. Commun. 10, 720 (2019).

26. Roberts, S. A. et al. An APOBEC cytidine deaminase mutagenesis pattern is widespread in human cancers. Nat. Genet 45, 970-976 (2013).

27. Haradhvala, N. J. et al. Mutational strand asymmetries in cancer genomes reveal mechanisms of DNA damage and repair. Cell 164, 538-549 (2016).

28. Bhagwat, A. S. et al. Strand-biased cytosine deamination at the replication fork causes cytosine to thymine mutations in Escherichia coli. Proc. Natl Acad. Sci. USA 113, 2176-2181 (2016)

29. Hoopes, J. I. et al. APOBEC 3 A and APOBEC3B preferentially deaminate the lagging strand template during DNA replication. Cell Rep. 14, 1273-1282 (2016).

30. Seplyarskiy, V. B. et al. APOBEC-induced mutations in human cancers are strongly enriched on the lagging DNA strand during replication. Genome Res. 26, 174-182 (2016).

31. Kumar, R. et al. HumCFS: a database of fragile sites in human chromosomes. BMC Genomics 19, 985 (2019).

32. Chan, K. et al. An APOBEC3A hypermutation signature is distinguishable from the signature of background mutagenesis by APOBEC3B in human cancers. Nat. Genet. 47, 1067-1072 (2015).

33. Burns, M. B. et al. APOBEC3B is an enzymatic source of mutation in breast cancer. Nature 494, 366-370 (2013).

34. Burns, M. B., Temiz, N. A. \& Harris, R. S. Evidence for APOBEC3B mutagenesis in multiple human cancers. Nat. Genet. 45, 977-983 (2013).

35. Kazanov, M. D. et al. APOBEC-induced cancer mutations are uniquely enriched in early-replicating, gene-dense, and active chromatin regions. Cell Rep. 13, 1103-1109 (2015).

36. Sharma, S., Patnaik, S. K., Kemer, Z. \& Baysal, B. E. Transient overexpression of exogenous APOBEC3A causes C-to-U RNA editing of thousands of genes. RNA Biol. 14, 603-610 (2017).

37. Bommarito, S., Peyret, N. \& SantaLucia, J. Jr. Thermodynamic parameters for DNA sequences with dangling ends. Nucleic Acids Res. 28, 1929-1934 (2000).

38. Yakovchuk, P., Protozanova, E. \& Frank-Kamenetskii, M. D. Base-stacking and base-pairing contributions into thermal stability of the DNA double helix. Nucleic Acids Res. 34, 564-574 (2006).

39. Bao, L., Zhang, X., Jin, L. \& Tan, Z. Flexibility of nucleic acids: from DNA to RNA. Chin. Phys. B 25, 018703 (2015).

40. Bastin-Shanower, S. A. \& Brill, S. J. Functional analysis of the four DNA binding domains of replication protein A. The role of RPA2 in ssDNA binding. J. Biol. Chem. 276, 36446-36453 (2001).

41. Kim, C., Paulus, B. F. \& Wold, M. S. Interactions of human replication protein A with oligonucleotides. Biochemistry 33, 14197-14206 (1994).

42. Kouno, T. et al. Crystal structure of APOBEC3A bound to single-stranded DNA reveals structural basis for cytidine deamination and specificity. Nat. Commun. 8, 15024 (2017).

43. Muhire, B. M. et al. Evidence of pervasive biologically functional secondary structures within the genomes of eukaryotic single-stranded DNA viruses. J. Virol. 88, 1972-1989 (2014).
44. Hess, J. M. et al. Passenger hotspot mutations in cancer. Cancer Cell. 36, 288-301.e14 (2019).

45. Kolesnikova, S. et al. GTP-dependent formation of multimeric Gquadruplexes. ACS Chem. Biol. 14, 1951-1963 (2019).

46. Svehlova, K., Lawrence, M. S., Bednarova, L. \& Curtis, E. A. Altered biochemical specificity of G-quadruplexes with mutated tetrads. Nucleic Acids Res. 44, 10789-10803 (2016).

47. Buisson, R., Lawrence, M. S., Benes, C. H. \& Zou, L. APOBEC3A and APOBEC3B activities render cancer cells susceptible to ATR inhibition. Cancer Res. 77, 4567-4578 (2017).

48. Stenglein, M. D., Burns, M. B., Li, M., Lengyel, J. \& Harris, R. S. APOBEC3 proteins mediate the clearance of foreign DNA from human cells. Nat. Struct. Mol. Biol. 17, 222-229 (2010).

49. SantaLucia, J. Jr. A unified view of polymer, dumbbell, and oligonucleotide DNA nearest-neighbor thermodynamics. Proc. Natl Acad. Sci. USA 95, 1460-1465 (1998).

50. Ellrott, K. et al. Scalable open science approach for mutation calling of tumor exomes using multiple genomic pipelines. Cell Syst. 6, 271-281 e277 (2018).

51. Langenbucher, A. et al. An extended APOBEC3A mutation signature in cancer. https://doi.org/10.5281/zenodo.4456072 (2021).

\section{Acknowledgements}

We thank Reuben Harris for the APOBEC3A/B antibody obtained through the NIH AIDS Reagent Program, Division of AIDS, NIAID, NIH. This work is supported by NIH grant CA249291 (M.S.L.), CA212154 (R.B.), CA218856 (L.Z.), and AI144708 (A.S.B.). R.B. is supported by the California Breast Cancer Research Program, the Concern foundation, and a University of California Cancer Research Coordinating Committee (CRCC) award grant. The Chao Family Comprehensive Cancer Center is supported by a core grant P30CA062203. M.S.L. is supported by startup funds from the MGH Center for Cancer Research.

\section{Author contributions}

A.L., R.B., and M.S.L. conceived and designed the study. A.L., J.F.W., and M.S.L. performed the bioinformatic analyses of APOBEC mutation signatures in patient tumors. D.B. and E.B. performed the biochemical experiments with cancer cell lines and in vitro APOBEC activity assays. R.S. performed uracil pull-down experiments and analyzed the sequencing of bacterial genomic DNA. A.L. wrote the manuscript. L.Z., A.S.B., R.B., and M.S.L. supervised the study and contributed to writing and editing the manuscript.

\section{Competing interests}

The authors declare no competing interests.

\section{Additional information}

Supplementary information The online version contains supplementary material available at https://doi.org/10.1038/s41467-021-21891-0.

Correspondence and requests for materials should be addressed to L.Z., A.S.B., R.B. or M.S.L.

Peer review information Nature Communications thanks James Alvarez, Hidewaki Nakagawa and the other, anonymous, reviewer(s) for their contribution to the peer review of this work. Peer reviewer reports are available.

Reprints and permission information is available at http://www.nature.com/reprints

Publisher's note Springer Nature remains neutral with regard to jurisdictional claims in published maps and institutional affiliations.

Open Access This article is licensed under a Creative Commons Attribution 4.0 International License, which permits use, sharing, adaptation, distribution and reproduction in any medium or format, as long as you give appropriate credit to the original author(s) and the source, provide a link to the Creative Commons license, and indicate if changes were made. The images or other third party material in this article are included in the article's Creative Commons license, unless indicated otherwise in a credit line to the material. If material is not included in the article's Creative Commons license and your intended use is not permitted by statutory regulation or exceeds the permitted use, you will need to obtain permission directly from the copyright holder. To view a copy of this license, visit http://creativecommons.org/ licenses/by/4.0/.

(C) The Author(s) 2021 